1 Evidence for the 'safety in density' effect for cyclists; validation of agent-based

3

4 5

6

7

8

9

10

11

12

13

2

3

\title{
modelling results
}

\author{
Dr Jason Thompson ${ }^{1,2}$, Dr Jasper S. Wijnands ${ }^{1}$, Dr Suzanne Mavoa ${ }^{3}$, Katherine \\ Scully ${ }^{1}$, Professor Mark Stevenson ${ }^{1}$ \\ ${ }^{1}$ University of Melbourne, Transport, Health and Urban Design Research Hub, \\ University of Melbourne, Australia, Ph +61 383447782 \\ ${ }^{2}$ University of the Sunshine Coast, Human Factors and Socio-Technical Systems \\ Research Unit \\ ${ }^{3}$ University of Melbourne, School of Population and Global Health \\ Word Count: 3029
}


16 The safety in numbers ( $\mathrm{SiN}$ ) effect for cyclists is widely observed but remains

17 poorly understood. Although most studies investigating the SiN phenomenon have 18 focused on behavioural adaptation to 'numbers' of cyclists in the road network, 19 previous work in simulated environments has suggested SiN may instead be driven 20 by increases in local cyclist spatial density, which prevents drivers from attempting 21 to move through groups of oncoming cyclists. This study therefore set out to 22 validate the results of prior simulation studies in a real-world environment. Time23 gap analysis of cyclists passing through an intersection was conducted using five 24 hours of video-observation of a single intersection in the city of Melbourne, 25 Australia, where motorists were required to 'yield' to oncoming cyclists. Results 26 demonstrated that potential collisions between motor-vehicles and cyclists reduced 27 with increasing cyclists per minute. These results successfully validate those 28 observed under simulated conditions, supporting evidence of a proposed causal 29 mechanism related to safety in density (SiD) rather than safety in numbers, per se. 30 Implications of these results for transportation planners, cyclists, and 31 transportation safety researchers are discussed, suggesting that increased cyclist 32 safety could be achieved through directing cyclists toward focused, strategic corridors rather than dispersed across a network. 
- It is understood that greater numbers of cyclists in a road transport system reduces risk per cyclist, producing a 'safety in numbers' effect

- Potential mechanisms underlying the safety in numbers effect are contested

- A candidate causal mechanism underlying the Safety in Numbers effect has been suggested based on local spatial density of cyclists; Safety in Density.

\section{What this study adds}

- This work provides empirical validation of prior simulation results conducted using agent-based modelling.

- Higher-density groups of passing cyclists prevent potential collisions between motor-vehicles who reject available time-gaps.

- This mechanism provides insight into the SiN phenomenon, which has so far eluded comprehensive explanation. 


\section{Introduction}

48 The safety in numbers (SiN) effect for cyclists is a widely referenced and observed, 49 but poorly understood phenomenon[1-3]. Although a wide range of academic and 50 applied studies cite $\mathrm{SiN}$ as a potential solution to car vs cyclist crashes (hereby 76 referred to as 'collisions')[3-7], there is currently little definitive evidence to guide policy-makers or transport planners in how to use SiN to create a safer cycling environment beyond simply encouraging 'more cyclists' into the system. Despite the lack of evidence surrounding the mechanism(s) that underpin it, the most widely forwarded understanding of $\mathrm{SiN}$ is that it is driven by "behavioural adaptation' among drivers[8,9]. That is, the more drivers see and become aware of cyclists on the road (or become cyclists, themselves), the more they learn to drive safely around them. Other authors have suggested that variation in risk associated with behavioural adaptation may be supplemented or off-set by improvements to cycling infrastructure[10], seasonal characteristics, [6] general traffic characteristics[11, 12], or behaviour of cyclists[13] that coincide with changes in cycling rates.

There is little doubt that the SiN effect is genuine. Observed at both a macro and meso-level, there is consistency across studies that the per capita number of collisions between cyclists and cars (i.e., collision risk) for cyclists increases more slowly as more cyclists enter the system[3]. However, beyond manifold studies that have used historic datasets of collisions and cyclist volumes to demonstrate this effect, as well as more recent work involving video-recording of intersections across seasons[6], there is little research studying car vs cyclist interactions at the individual (micro) level. This has restricted the ability of studies to draw conclusions regarding the mechanisms underlying $\mathrm{SiN}$ that extend much beyond conjecture. It is important to address this knowledge gap as proposed interventions designed to reduce deaths and injuries among on-road cyclists may prove ineffective, or potentially more dangerous, without accurate understanding.

Recognising these limitations, previous work has attempted to create agent-based models (ABMs) of cyclist vs car interactions within simulated transport systems[14-16]. ABMs allow both macro and micro-level behaviour of systems to 
80

be observed. Within such models, the behaviour of individual drivers and cyclists can be monitored alongside the overall performance (i.e., safety) of the system.

The results of ABMs of car vs cyclist interactions have suggested that the SiN effect can be replicated in simulated transport systems where drivers demonstrate behavioural adaptation in response to exposure to cyclists[15, 16]. However, it can also be replicated in circumstances where drivers show no capacity to 'learn' to drive safely around cyclists at all[14]. Rather, the SiN effect can be reproduced simply through encouraging behaviour that leads to the formation of higher-density cyclist groups. This reduces the surface-area to volume ratio of cyclists to cars in a manner analogous to the 'selfish-herd' mechanism common to biological predatorprey relationships[17, 18]. The previous computational observations and synthetic evidence[19] produced by these ABMs do not discount the fact that behavioural adaptation may be an additional mechanism contributing to the SiN effect, but suggests that behavioural adaptation is not a necessary condition for reducing risk given certain spatial configurations of cyclists in a network. Therefore, rather than SiN, the theory informed by agent-based modelling proposes that higher levels of safety can be achieved through increasing density; i.e., safety in density (SiD).

While acknowledged as a potential mechanism[9], the SiD hypothesis has been criticised from the perspective that it is derived from computational models, only, and that no in-situ empirical evidence exists that cyclists 'cluster' in the real world. Whilst the same concerns regarding lack of empirical evidence can also be applied to the behavioural adaptation hypothesis, it is somewhat simpler to address concerns related to spatial phenomena, which can be directly observed.

The focus of this study was therefore to observe micro-level interactions of cyclists and vehicles at an intersection mirroring that created in prior ABMs to determine how cyclist density is associated with per-capita collision risk for cyclists. In doing so, we aimed to gather empirical evidence of the SiD hypothesis' operation in a real-world situation under conditions that prevented opportunity for behavioural adaptation. Consistent with previous macro-level research demonstrating a decreasing risk of collision per cyclist with increasing cyclist volume, it is hypothesised that increasing density of cyclists at a single intersection will be 
associated with a decreasing per-capita cyclist risk of potential collision with cars when interacting in 'yield' situations.

116

\section{Method}

\section{Intersection characteristics}

119 Data collection occurred through video recording of naturalistic traffic behaviour occurring at an inner-city cross-intersection in Melbourne, Australia. The intersection was chosen for four reasons. Firstly, it was located along a popular cycling route that featured a marked (not separated) cycling lane carrying a high volume of commuter cyclists to and from Melbourne's central business district. This ensured that adequate volume of cyclist and car interactions could be logged and analysed within the recording period. Secondly, because the north-south route on which cyclists travelled was largely blocked to car traffic, the primary movement of cars at the intersection at the recording time was across the intersection in an East-West direction, producing consistent required judgements and behaviours among drivers. Thirdly, because the selected intersection has previously recorded several car vs cyclist collisions, it has been identified as 'high risk' by local road authorities to the extent that it features highvisibility electronic, flashing signs that warn motorists that cyclists are present (see

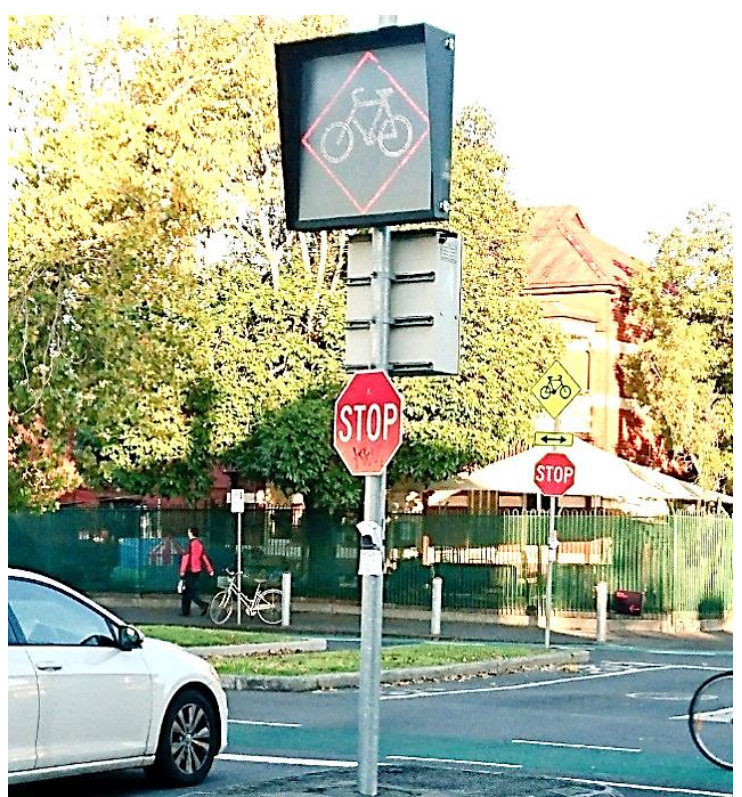

Figure 1). In this respect, all drivers approaching the intersection were provided with standardised information designed to raise awareness that cyclists were potentially present. Lastly, the combination of intersection and vehicle characteristics described above, were like those observed in the previously analysed simulated environments [i.e., 14, 20, 21]. 
138 Figure 1. The selected intersection (pictured) was chosen based on high cyclist 139 volume, consistency of car movements, and standardised information regarding 140 the possible presence of cyclists provided through warning signs.

\section{Video recording of car and cyclist interactions}

142 Video recording occurred over a period of five separate days over an 8-day period 143 during daylight hours between 8am and 9am during early June, 2017 to capture 144 morning commuter traffic. Both a variety of days of the week and weather were 145 sampled including $2 \times$ Mondays, a Wednesday, Thursday, and a Friday. One day of the 146 week (Tuesday) was not captured due to heavy rain, making recording impractical. A 147 small, concealed video camera was mounted in a tree on the median strip facing 148 North-East at an angle of approximately 45 degrees to the centre of the intersection.

149 This provided full view of oncoming Northern and Eastern traffic as well as cross150 traffic from the West. A view of the selected intersection and indicative camera angle 151 is shown in Figures 2 and 3. Though the camera was concealed so as not to distract 152 drivers or cyclists, researchers remained close to the intersection to answer questions 153 from pedestrians or residents who may have noticed the researchers mounting and 154 un-mounting the cameras at the beginning and end of each recording session. The 155 research was approved by the University of Melbourne Human Research Ethics 156 Committee (1749212.1).

157

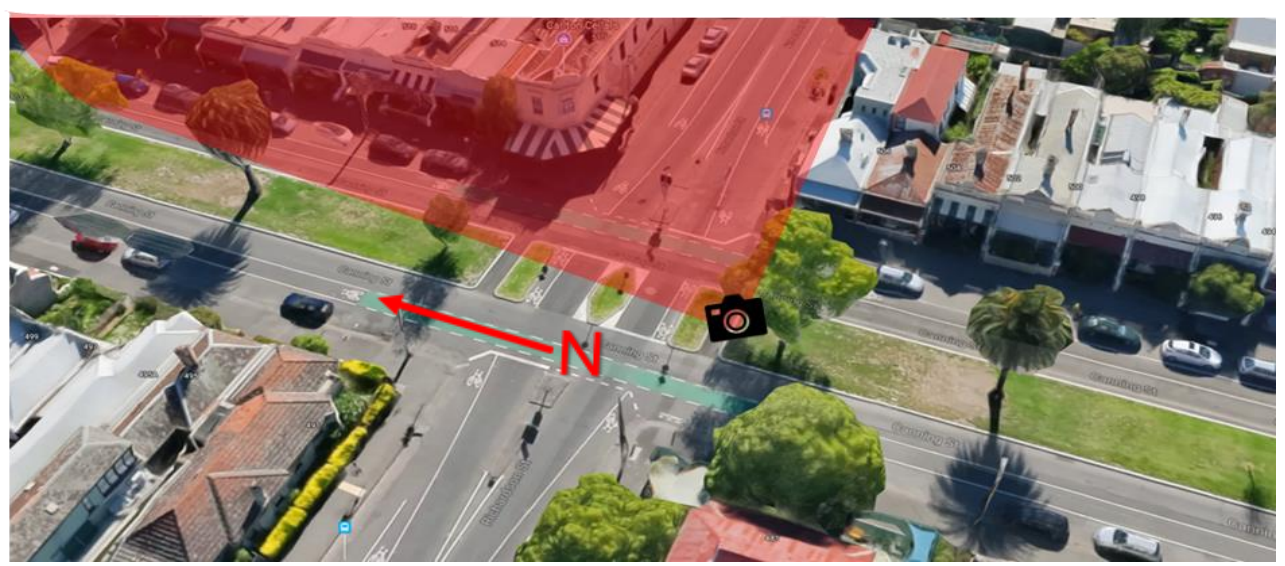

159 Figure 2. View of the selected intersection showing orientation, location and 160 coverage of the video camera. 
Time-stamped video footage was manually coded to an accuracy of $0.1 \mathrm{sec}$ by the research team (including review of coding accuracy) for all cyclist movements flowing North-South and car-movements flowing East-West (see Figure 3). All other car, pedestrian and bicycle movements were ignored for the purposes of this study. Instances where other vehicle movement potentially interfered with bicyclevehicle interactions (e.g., a North-South travelling car entered the intersection) were also removed from analysis. The following data was coded for each cyclist as they moved through the intersection:

- cyclist unique identification number (identified per event, not by person);

- time at which the cyclist moved through the centre of the intersection; and

- time-gap between the leading cyclist to the following cyclist.

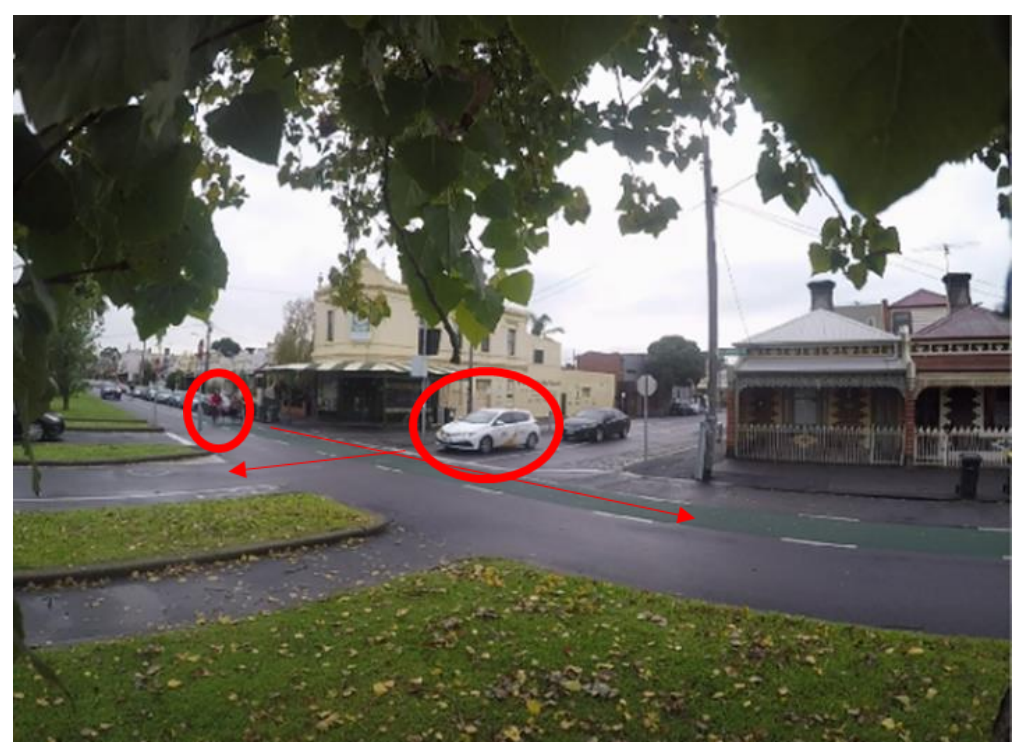

Figure 3. View from the concealed camera of the intersection including a cyclist approaching from the North and two waiting drivers from the East.

Similarly, the following data was recorded for each driver movement:

- driver unique identification number (unrelated to vehicle license plate);

- time at which the driver arrived at the intersection and was at the front of the queue; and

- time at which the driver started crossing the intersection.

Recording these variables produced a temporally ordered list of cyclists and drivers that moved through the intersection over five, 1-hour periods. This allowed calculation of variables, including: 
192

193 Potential collisions were recorded as instances in which a gap of $<4.2$ seconds
- the time gap of approaching cyclists to the intersection at the instant when drivers arrived at the intersection (sec);

- number of cyclists each driver gave way to before moving away from the intersection ( $n)$;

- time gap between each cyclist passing through the intersection (sec); and minute (cyclists / $\min$ ).
- frequency of cyclists passing through the intersection during each recorded cyclist then passing through. Potential collisions therefore represented instances in which drivers made a judgement that was beneath the $50^{\text {th }}$ percentile of that left by all drivers, leading them to potentially misjudge the distance or time in which it would take them to clear the intersection before the arrival of a cyclist (incorrect gap acceptance)[22]. The selection of the $<4.2$ second threshold for categorisation of potential collisions was made after analysis of gap acceptance and rejection distributions detailed in the Results section, below. Though $50 \%$ of drivers rejected gaps below 4.2 seconds (i.e., waited), these instances were considered of negligible risk [23] or 'missed opportunities' [22] by the driver.

\section{Results}

Figure 4 shows two kernel density estimates [24] of the gap acceptance of drivers who reject (green) or accept the time gap (yellow) upon arrival at the intersection. The distributions overlap, with gaps below 10 seconds generally those when drivers became more likely to 'reject' the time-gap as too small for entry (observed: [0.1, 12.4]) and longer gaps ( $>10$ seconds) when drivers accepted the gap and moved through the intersection (observed: $[2.3,45.7]$ ). 
213

214

215

216

217 Of all observed drivers, 489 rejected the gap (waited), while 431 cars accepted the 218 gap (did not wait). If drivers waited for cyclists at all, the mean number of bicycles 219 they gave way to was 2.31 (SD 2.11). The probability that a driver accepted or 220 221 222 223 224 225 226 227 228

Figure 4. Time gap acceptance (yellow) or rejection (green) for drivers arriving at the intersection and arrival of approaching cyclists. rejected the time gap until the next cyclist reached the intersection is shown in Figure 5. This figure shows a critical time gap[25] of approximately 4.2 seconds at which half of the drivers decided to wait, while the other half did not wait. At this interval, cyclists experienced the most uncertain driver behaviour. The observed critical time gap was in the mid-range of previously modelled acceptance ranges of between 2 and 6 seconds [23]. Note that the probability of a driver waiting did not monotonically decrease for increasing gap times, potentially due to the limited observational data in this research.

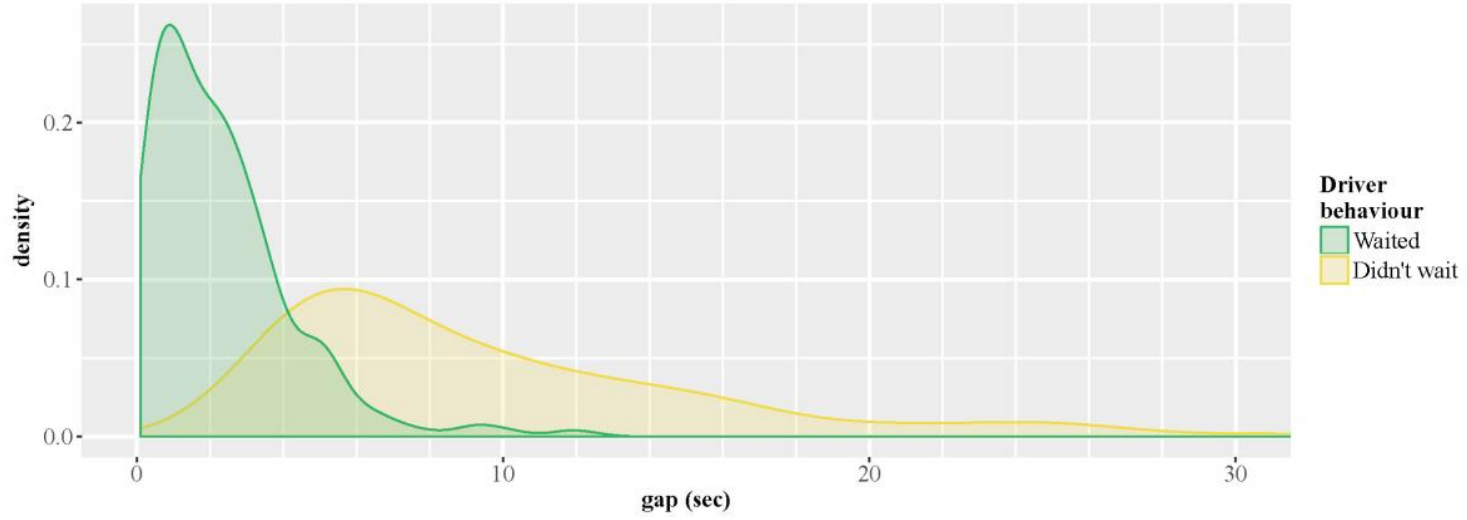




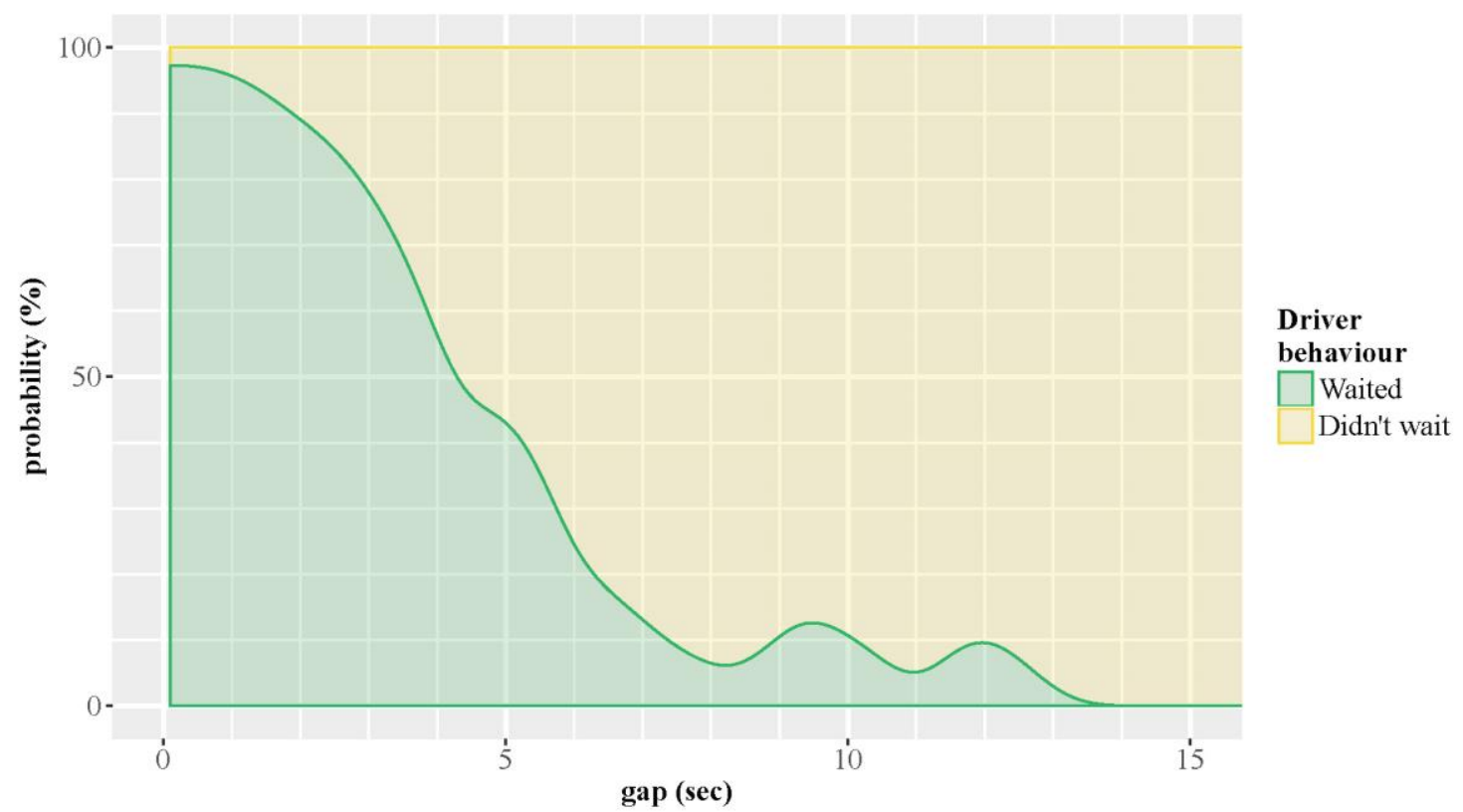

gap (sec)

230 Figure 5. Probability estimates for driver behaviour based on the time gap to 231 the next cyclist showing proportions of time-gap acceptance (yellow) and 232 rejection (green).

\section{Collision risk with variation in cyclist density}

The frequency of potential collisions per minute $(y)$ (accepted time gaps of $<4.2$ seconds) was modelled using the number of cyclists per minute $(x)$ as a single explanatory variable. Potential collisions and the number of cyclists were counted each minute across the total five hours of captured video footage, resulting in an appropriate sample of cyclist density and potential collision counts $(n=385)$. Count data was not affected by over-dispersion $(p=0.99)$ [26]. Hence, the potential collision count was modelled using Poisson regression[e.g., 27] following Equation 242 (1).

$$
y=e^{a+\beta x}+\varepsilon
$$

244 Parameter estimates are presented in Table 1 and are significant at a confidence

245 level $<0.001$. The fitted number of potential collisions is then divided by the

246 number of cyclists to obtain the per cyclist collision risk at each level of cyclist

247 density (see Figure 6). Hence, the potential collision count was modelled using

248 Poisson regression[e.g., 27] following Equation (1), with $\alpha$ and $\beta$ the model's

249 parameters related to the intercept and sensitivity to the number of cyclists and $\varepsilon$

250 the random error component. 
Table 1. Parameter estimates of Poisson regression.

\begin{tabular}{lllll}
\hline & Estimate & Std. error & $\boldsymbol{z}$ value & $\mathbf{P}(>|z|)$ \\
\hline$\alpha$ & -0.32 & 0.10 & -3.31 & .000 \\
$\beta$ & 0.07 & 0.01 & 6.65 & .000 \\
\hline
\end{tabular}

253

254 The resulting estimated risk per cyclist $(r)$ was:

255

$$
r=\frac{e^{-0.32+0.07 x}}{x}
$$

256

259

260 Evident from Figure 6 is the significant reduction in potential per-cyclist crash risk 261 in circumstances of higher-density cycling. That is, in periods where more cyclists 262 passed through the intersection per minute, per-cyclist potential collision risk 263 264 265

number of cyclists per minute

Figure 6. Potential per-cyclist crash risk (y-axis) with increasing count of cyclists per minute (x-axis). decreased. This effect of cyclist density on potential collision risk was independent of behavioural adaptation by drivers.

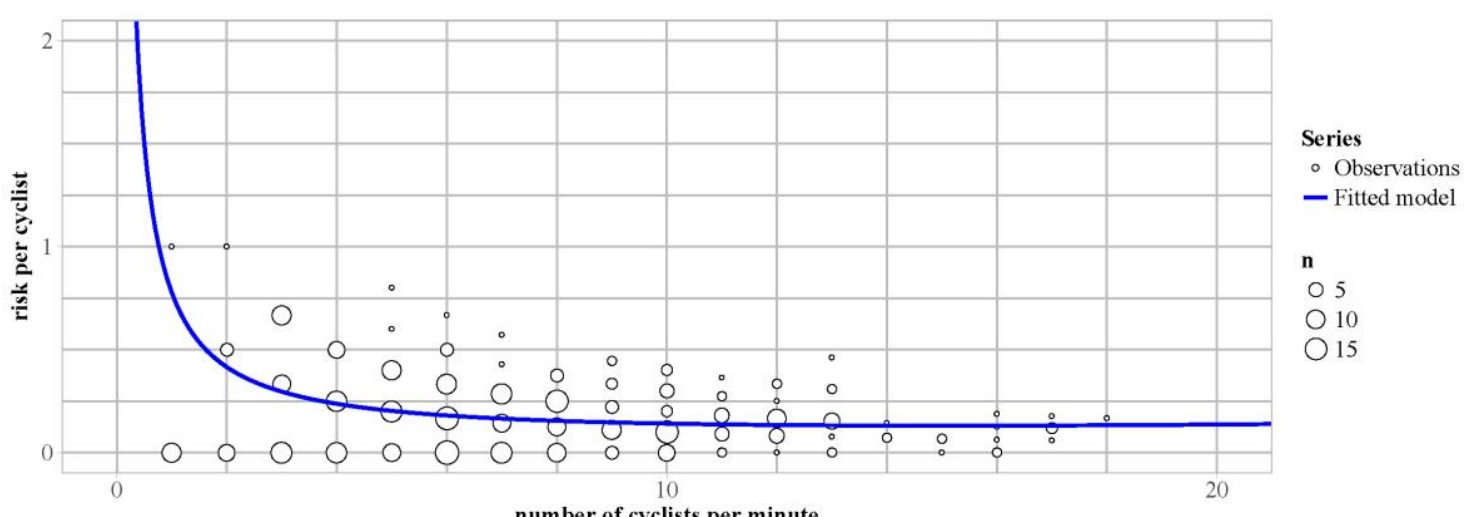




\section{Discussion}

267 The current study supported our hypothesis, demonstrating that potential per268 cyclist collision risk decreased with increasing cyclist density (i.e., cyclist count per 269 minute). It demonstrates the SiD hypothesis' operation in a real-world situation 270 under conditions where observations occurred within a limited period, precluding opportunity for behavioural adaptation by drivers. Extrapolated across a transport network, these findings are consistent with previous macro-level observations relating to the $\mathrm{SiN}$ effect. However, they make an important advance by providing

274 further insight into a potential mechanism underlying the reduction in per-cyclist 275 risk, namely, cyclist density.

276

277 In a series of ABMs, Thompson et al.[14-16] demonstrated that the SiN effect could 278 be reproduced within a synthetic road network with or without the influence of 279 modelled behavioural adaptation among simulated drivers. This indicated that SiN

280

281

282

283

284

285

286

287

288

289

290

291

292

293

294

295

296

297 may be better expressed as SiD because changes in the observed per-capita risk among simulated cyclists was driven by a spatial phenomenon primarily related to the density of cyclists in the system rather than numbers, per se. However, in a subsequent review of potential mechanisms underlying SiN, Jacobsen et al. (2015) discounted the likelihood of this candidate mechanism, arguing that no empirical evidence of SiD had been demonstrated outside computational models. This study now provides such evidence, shedding potentially new light on mechanisms underpinning an issue that has interested transportation safety researchers for some time [e.g., 1, 2, 12]. For example, in a recent exploration and meta-analysis, Elvik[12] questioned the causal mechanisms underlying SiN by highlighting an apparently counter-intuitive relationship between cyclist volume and the strength of the safety in numbers effect. Elvik noted that it was weakest when cycling volumes were highest and that no clear relationship existed between the strength of the SiN effect and the ratio of motor-vehicles to cyclists across a network. Viewed as a phenomenon that is the result of an aggregation of local level interactions, however, we consider that Elvik's findings are consistent with results presented here.

298 That is, at specific times and locations where cyclist density is high, the movement of motor-vehicles (and potential collisions) is restricted because time gaps 
between cyclists are smaller and more likely to be rejected by drivers. At specific locations and times when cyclist density is low, time-gaps are larger and more likely to be accepted by drivers, resulting in higher per-capita collision risk as drivers and cyclists more frequently interact. These interactions considered at a micro-level may be independent of total cyclist numbers, total motor-vehicle numbers, or motor-vehicle to cyclist ratios when measured across the network[14, 16]. When total cyclists, motor-vehicles, and collisions are then measured at the level of a region, city, or country, cyclist numbers vs reduced crash risk profiles may well emerge. However, it does not necessarily follow that cyclist numbers or ratios are the causal mechanism underlying SiN. Rather, higher cyclist numbers may simply make it more likely that locations of dense cycling activity occur within a network, which restricts unsafe interactions between cars and cyclists in those locations and results in reduced overall crash risk per-cyclist.

Consistent with reduction in strength of the SiN effect at high cyclist or pedestrian volumes as reported by Elvik[12], the SiD effect may also be subject to saturation at very high volumes when gap rejection tolerances for individual drivers[23] are exceeded. This can be seen in Figure 6, where very little reduction in risk is achieved for cyclists in circumstances where the per-minute rate exceeds 12 cyclists per minute (an average time-gap of 5 seconds). Conversely, if cyclist numbers are dispersed widely across a network, time-gaps may be wider, more likely to be accepted by drivers, and the opportunity for the $\mathrm{SiD}$ mechanism to operate may be reduced[14]. Again, this is not dissimilar to the selfish-herd mechanism observed in biological systems, which protects individual herdmembers from predators by reducing the surface-area to volume ratio of the group $[17,18]$. Dense groups of cyclists have a smaller surface-area to volume ratio than dispersed groups, which provides a protective effect against motor-vehicle incursions through the group.

The policy implications of these findings are important to consider for transportation planners, cyclists, and researchers, alike. For planners, they suggest that reducing risk of collision for cyclists in 'yield' situations can be achieved through increasing the density of cyclists along strategic routes. This could be achieved through identifying a well-connected network of cycling routes that are 
highlighted and recommended to cyclists. Cyclists, themselves, can be encouraged to use these routes in the knowledge that higher density cycling that reduces the time-gap between themselves and other cyclists (to less than 4.2 seconds in the case of the observed intersection) may reduce car vs cyclist crash risk for themselves and fellow travellers. This is a departure from previous policy advice that has advocated that decreased crash risk can be achieved by increasing number of cyclists across the network without consideration of cyclists' immediate local context[e.g., 5, 28].

342

343 For researchers, these results highlight the utility of ABMs to identify new

344 theoretical models in-silico that can then be tested in the field. To date, most work 345 attempting to study SiN has been based on cross-sectional or longitudinal 346 observations of crash-numbers alongside cyclist and motor-vehicle volumes[3, 11].

347 The availability of aggregate data in this form originally drove the development of 348 the SiN theory[4]. However, it is possible that the strength of the original findings 349 may have led to a subsequent degree of observer dependence, whereby data 350 collection has largely continued to follow existing theory focused on 'numbers' of cyclists across a system rather than alternative spatial mechanisms as described

352 here. Instead, the synthetic evidence collected from ABMs[14-16] has enabled a 353 new perspective on the way we could collect observational data[29] related to cycling safety that has been operationalised in this study.

356 There are limitations to the present research that restrict generalisation of results.

357 Firstly, the studied interactions between drivers and cyclists were of purposefully designed, simple, select nature where only 'give-way' ('yield') behaviour by drivers was observed. Reductions in potential collision risk in situations where cyclists travelled in parallel with (i.e., alongside) drivers were not studied. Further, though

361 the density measure used in this study relates to time-gaps between cyclists and between cyclist and car-movements, the actual distance in metres was not recorded. It is therefore unclear whether drivers' judgement on when to move

364 through the intersection was based on an estimate of time (e.g., estimation of 365 distance $\times$ speed)[22] or simply distance from the cyclist; although it seems 366 reasonable to assume that drivers incorporate the estimated speed of the cyclist (i.e., time-based reasoning). 
369 Another limitation is that the cyclist / motor vehicle interaction under study related 370 to driver judgement and behaviour of West-travelling motor-vehicles, only. 371 However, during observation, five near-misses and one minor crash were also 372 witnessed for drivers travelling East, who failed to stop for oncoming cyclists at all.

373 Each of these near-miss incidents required cyclists to take evasive action (i.e., to 374 brake heavily) and occurred in circumstances where drivers appeared to be blinded 375 to on-coming cyclists by bright, early-morning sunshine (see Figure 7). The SiD 376 model as described here is unable to account for these 'failed to see'[30] 377 occurrences where saliency of cyclists was either low or the driver's perception was 378 poor. Previous theoretical models have, however, attempted to account for these 379 factors in the context of the SiD effect[16], providing an avenue for future 380 observational studies to explore this issue. This highlights a final important 381 limitation in that our methodology did not measure counts of actual collisions, but 382 situations in which a collision was made more possible through a potentially risky 383 driver manoeuvre. It therefore assumes that the ratio of actual collisions to 384 potential collisions as defined here is robust.

385

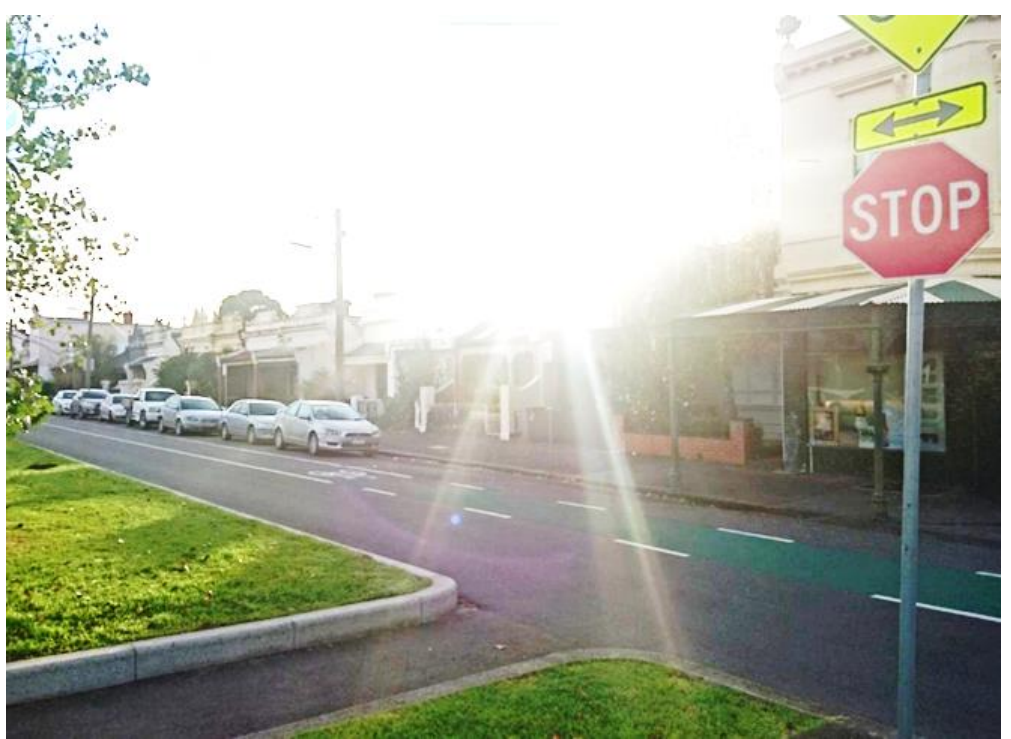

387 Figure 7. Photograph taken from the intersection facing East, showing similar circumstances under which 5 near-misses and one minor collision were observed, possibly due to drivers being 'blinded' to the presence of cyclists by early-morning sunshine.

\section{Conclusions}


393 The current study has provided empirical validation of the SiD hypothesis' 394 operation in a real-world situation. It has demonstrated that reduced potential 395 collision risk is associated with reduced time-gaps between cyclists passing

396 through intersections that prevent motor-vehicles from moving between on-coming 397 cyclists (gap rejection). Using a methodology based on synthetic evidence gathered 398 evidence from previous computational observation and experimentation[19-21, 31], 399 this work has provided further support for a candidate causal mechanism 400 underlying the widely observed general relationship between cycling numbers and 401 safety; a mechanism that has thus far eluded comprehensive explanation in the 402 cycling safety literature.

403

404 Acknowledgements

405 Dr Jason Thompson is supported by an Australian Research Council Discovery Early 406 Career Research Award. Dr Suzanne Mavoa is supported by an NHMRC Early 407 Career Fellowship (\#1121035). Professor Mark Stevenson is supported by an 408 NHMRC Fellowship (\#1043091). 
410 1. Bhatia, R. and M. Wier, "Safety in Numbers" re-examined: can we make valid or practical inferences from available evidence? Accid Anal Prev, 2011. 43(1): p. 235-40.

2. Christie, N. and I. Pike, Is it safe in numbers? Inj Prev, 2015. 21(4): p. 276-7.

3. Elvik, R. and T. Bjørnskau, Safety-in-numbers: A systematic review and metaanalysis of evidence. Safety Science, 2017. 92: p. 274-282.

4. Jacobsen, P.L., Safety in numbers: more walkers and bicyclists, safer walking and bicycling. Inj Prev, 2003. 9(3): p. 205-9.

5. Robinson, D.L., Safety in numbers in Australia: more walkers and bicyclists, safer walking and bicycling. Health Promot J Austr, 2005. 16(1): p. 47-51.

6. $\quad$ Fyhri, A., et al., Safety in numbers for cyclists-conclusions from a multidisciplinary study of seasonal change in interplay and conflicts. Accid Anal Prev, 2016.

7. Tin, S.T., et al., Regional variations in pedal cyclist injuries in New Zealand: safety in numbers or risk in scarcity? Aust N Z J Public Health, 2011. 35(4): p. 357-63.

8. Beanland, V., M.G. Lenné, and G. Underwood, Safety in numbers: Target prevalence affects the detection of vehicles during simulated driving. Attention, Perception and Psychophysics, 2014. 76(3): p. 805-813.

9. Jacobsen, P.L., D.R. Ragland, and C. Komanoff, Safety in Numbers for walkers and bicyclists: exploring the mechanisms. Inj Prev, 2015. 21(4): p. 217-20.

10. Reynolds, C.C., et al., The impact of transportation infrastructure on bicycling injuries and crashes: a review of the literature. Environ Health, 2009. 8: p. 47.

11. Aldred, R., et al., Contextualising Safety in Numbers: a longitudinal investigation into change in cycling safety in Britain, 1991-2001 and 2001-2011. Injury Prevention, 2017.

12. Elvik, R., Exploring factors influencing the strength of the safety-in-numbers effect. Accident Analysis and Prevention, 2017. 100: p. 75-84.

13. Fyhri, A., T. Bjørnskau, and A. Backer-Grøndahl, Bicycle helmets - A case of risk compensation? Transportation Research Part F: Traffic Psychology and Behaviour, 2012. 15(5): p. 612-624.

14. Thompson, J., G. Savino, and M. Stevenson, Reconsidering the safety in numbers effect for vulnerable road users: an application of agent-based modeling. Traffic Inj Prev, 2015. 16(2): p. 147-53.

15. Thompson, J., et al., Estimating the safety benefit of separated cycling infrastructure adjusted for behavioral adaptation among drivers; an application of agent-based modelling. Transportation Research Part F-Traffic Psychology and Behaviour, 2017. 49: p. 18-28.

16. Thompson, J., G. Savino, and M. Stevenson, A model of behavioural adaptation as a contributor to the safety-in-numbers effect for cyclists. Transportation Research Part a-Policy and Practice, 2016. 85: p. 65-75.

17. Morrell, L.J., G.D. Ruxton, and R. James, Spatial positioning in the selfish herd. Behavioral Ecology, 2011. 22(1): p. 16-22.

18. Hamilton, W.D., Geometry for the selfish herd. Journal of Theoretical Biology, 1971. 31(2): p. 295-311.

19. Davis, P.K., et al., Priority Challenges for Social and Behavioral Research and Its Modeling. 2018, Santa Monica, CA:: RAND Corporation.

20. Thompson, J., et al., Estimating the safety benefit of separated cycling infrastructure adjusted for behavioral adaptation among drivers; an application of agent-based modelling. Transportation Research Part F: Traffic Psychology and Behaviour, 2017. 49: p. 18-28.

21. Thompson, J., G. Savino, and M. Stevenson, A model of behavioural adaptation as a contributor to the safety-in-numbers effect for cyclists. Transportation Research Part A: Policy and Practice, 2016. 85: p. 65-75. 
22. Hunt, M., D. Harper, and C. Lie, Mind the gap: Training road users to use speed and distance when making gap-acceptance decisions. Accident Analysis \& Prevention, 2011. 43(6): p. 2015-2023.

23. Pollatschek, M.A., A. Polus, and M. Livneh, A decision model for gap acceptance and capacity at intersections. Transportation Research Part B: Methodological, 2002. 36(7): p. 649-663.

24. Scott, D.W., Multivariate density estimation: theory, practice, and visualization. 2015: John Wiley \& Sons.

25. Fitzpatrick, K., Gaps accepted at stop-controlled intersections. Transportation Fitzpatrick, K., Gaps accepted at stop-controll
Research Record, 1991. 1303: p. 103-112.

26. Cameron, A.C. and P.K. Trivedi, Regression-based tests for overdispersion in the Poisson model. Journal of econometrics, 1990. 46(3): p. 347-364.

27. Venables, W. and B. Ripley, Random and mixed effects, in Modern applied statistics with S. 2002, Springer. p. 271-300.

28. University of New South Wales A Virtuous Cycle: Safety In Numbers For Bicycle Riders. ScienceDaily, 2008.

29. Tracy, M., M. Cerda, and K.M. Keyes, Agent-Based Modeling in Public Health: Current Applications and Future Directions. Annu Rev Public Health, 2018. 39: p. 77-94.

30. Herslund, M.B. and N.O. Jorgensen, Looked-but-failed-to-see-errors in traffic. Accid Anal Prev, 2003. 35(6): p. 885-91.

31. Thompson, J., M. Stevenson, and G. Savino, Reconsidering the safety in numbers effect for vulnerable road users: An application of agent-based modelling. Traffic Injury Prevention, 2014. In Press. 